\title{
АВТОМАТИЗАЦИЯ ОРГАНИЗАЦИИ ВЗАИМОПРОВЕРКИ РАБОТ ОБУЧАЮЩИХСЯ С ИСПОЛЬЗОВАНИЕМ РАЗРАБОТАННОГО ПЛАГИНА ДЛЯ LMS MOODLE
}

\section{AUTOMATION OF ORGANIZATION OF MUTUAL VERIFICATION OF STUDENT WORK USING THE DEVELOPED PLUGIN FOR LMS MOODLE \\ N. Nasyrov \\ N. Gorlushkina \\ Yu. Valitova \\ P. Tartynskikh}

Summary. Mutual verification of student works is one of the forwardlooking and relevant educational approaches in the conditions of spreading and increasing the importance of distance and blended learning. The paper deals with the problem of the need to automate routine and time-consuming actions to organize mutual verification of student works.

The description of the implemented plugin for LMS Moodle, which automates the creation of course elements that can be used to organize mutual verification of students' work is provided as a solution. The plugin allows you to create individual tasks for trainees with automatic generation of task descriptions, evaluation criteria, examples of work based on previously submitted data. The ability to display statistical data on each student allows you to create individual tasks based on the results demonstrated in the execution of previously issued tasks. The implemented module allows you to reduce the time spent on creating tasks for mutual verification.

Keywords: automation, mutual verification, workshop activity, individualization of the educational process, Moodle.

\section{Введение}

B ызовы времени требуют поиска новых деятельных, развивающих форм, средств и методов обучения и контроля результатов обучения. Изменения должны позволить повысить эффективность формирования профессиональных компетенций, востребованных рынком труда, за счет новых способов усвоения,

\author{
Насыров Наиль Фаизович \\ Аспирант, инженер, Университет ИТМО \\ pasdel@mail.ru \\ Горлушкина Наталия Николаевна \\ К.т.н., дочент, С.н.с., доцент, Университет ИТМО \\ nagor.spb@mail.ru \\ Валитова Юлия Олеговна \\ К.п.н., доцент, Университет ИТМО \\ julijawal@gmail.com \\ тартынских Петр Сергеевич \\ Инженер, Университет ИТМО \\ tartynskikh.ps@yandex.ru
}

Аннотация. Взаимопроверка студенческих работ - один из наиболее перспективных и актуальных подходов к организации процесса обучения в условиях распространения и повышения значимости дистанционного и смешанного обучения. В работе рассматривается проблема необходимости автоматизации рутинных и требующих существенных временных затрат действий по организации взаимопроверки работ обучающихся.

B качестве решения приводится описание реализованного плагина для LMS Moodle, автоматизирующего создание элементов курсов, с помощью которых можно организовать взаимопроверку работ обучающихся. Разработанный плагин позволяет формировать индивидуальные задания для обучающихся с автоматической генерацией описания заданий, критериев оценивания, примеров выполнения работ на основе заранее предоставленных данных. Возможность отображения статистических данных по каждому обучающемуся позволяет создавать индивидуальные задания на основе результатов, продемонстрированных при выполнении ранее выполненных заданий. Разработанный плагин позволяет снизить временные затраты на создание заданий для взаимопроверки.

Ключевые слова: автоматизация, взаимопроверка, модуль семинар, индивидуализация процесса обучения, Moodle.

мышления и учебной деятельности, развитие познавательных сил и творческого потенциала студента.

Одним из современных интерактивных методов организации взаимодействия студентов является взаимная проверка ими результатов работы друг друга. Это позволяет повысить вовлеченность обучающихся в процесс обучения, предоставить им возможность сравнить 


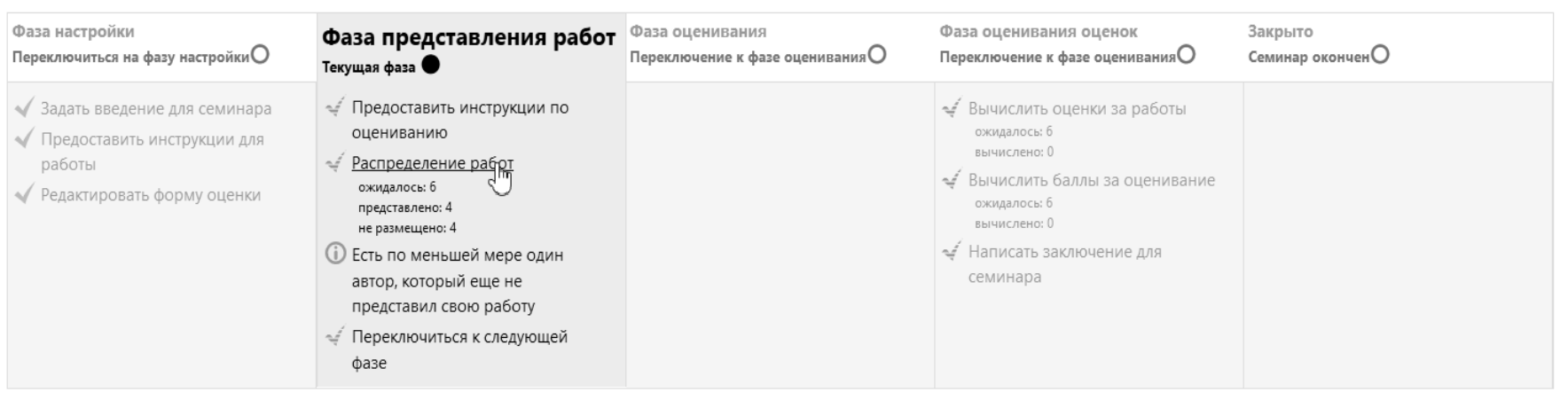

Рис. 1. Интерфейс страницы модуля Семинар

свои результаты с результатами других, оценить достаточность знаний для решения различных задач одного типа. Приемы взаимной проверки работ обучающимися при организации контроля получают все большее распространение во время занятий, развивая когнитивные, социальные, профессиональные навыки [1-4]. Оценивая работу сверстников, обучающиеся способны находить новые знания на основе имеющихся. Участвуя в проверке чужих работ, студент приобретает опыт оценочной деятельности, который пригодится в дальнейшей профессиональной деятельности. Это определяет все большую востребованность взаимопроверки в учебном процессе вузов.

В условиях увеличения доли смешанного и дистанционного обучения, наблюдаемого в последнее время, у преподавателей возникла проблема проверки студенческих работ со сложным результатом, которые требуют в большинстве дисциплин ручной длительной проверки преподавателем. Использование методов взаимопроверки позволяет преподавателям решить эту проблему.

Как отмечалось ранее, все активнее развиваются технологии дистанционного и смешанного обучения, в которые органично интегрируется обучение через системы дистанционного обучения (СДО, Learning management systems, LMS) и массовые открытые онлайн курсы (massive open online courses, MOOC) [5]. Этот факт подтверждается тем, что в разработку, наполнение контентом создаваемых курсов и развитие дистанционного обучения включились ведущие университеты по всему миру [6]. На текущий момент существует достаточное количество СДО. Однако, далеко не в каждой из них предусмотрен инструментарий взаимопроверки работ обучающихся.

В СДО, поддерживающих организацию взаимопроверки работ обучающихся, процесс создания подобных элементов курсов сопряжен со значительными временными затратами и выполнением однотипных, зачастую рутинных, действий. Очевидно, что процесс взаимопроверки в случаях, когда это можно осуществить без ущерба качеству процесса обучения, должен быть максимально автоматизирован.

Дальнейшее исследование проблемы автоматизации организации взаимопроверки и демонстрация полученного решения приводится на примере системы дистанционного обучения LMS Moodle. Выбор указанной системы в первую очередь обусловлен тем, что LMS Moodle имеет встроенный модуль Семинар (Workshop), предоставляющий функции организации взаимопроверки работ обучающимися. Стоит отметить, что в модуле реализована возможность использовать широкий спектр типов заданий по сложности, а также варьировать количество заданий в одном Семинаре.

Использованию LMS Moodle также способствовали такие факторы, как бесплатность системы, открытый исходный код, наличие локализации для русскоязычных пользователей, популярность в России [7].

\section{Применение LMS Moodle при взаимопроверке}

Для реализации взаимопроверки в LMS Moodle присутствует модуль курса Семинар, с помощью которого можно внедрить технологию взаимопроверки в процесс обучения. Семинар в LMS Moodle - это гибкий, функциональный модуль для проектирования такого вида занятий, где каждый студент не только выполняет собственную работу, но и оценивает результаты работы других студентов [8]. Однако, по мнению автора пособия [8], Семинары, предоставляя большое разнообразие вариантов использования, являются самым сложным элементом курса в настройке и управлении.

Для использования модуля Семинар определена последовательность действий, которую должны выполнить для планирования и управления процессом 
обучения преподаватель, а затем в процессе обучения и студенты. Эти действия можно иллюстрировать изображением страницы модуля в системе Moodle, представленном на рисунке 1.

Как видно из рисунка 1, в процессе взаимодействия с модулем Семинар выделяются пять фаз [9]. Рассмотрим фазы подробнее.

1. Фаза настройки. Происходит описание основных правил и критериев, которыми должны будут руководствоваться студенты. После чего преподаватель должен разместить задания, которые нужно будет выполнять студентам. При этом стоит отметить, что преподаватель может обозначить даты прохождения всех заданий студентами сразу, а в дальнейшем вносить коррективы в случае необходимости.

При создании Семинара необходимо устанавливать ряд параметров. В частности, следует указать стратегию оценивания (форму и методы оценивания), способ вычисления оценки за работу, оценку за оценивание, добавить инструкции для проверяющего, включая примеры выполнения работы. Кроме этого, необходимо отметить настройки обязательности загрузки файлов выполненной работы для проверки, указать теги, количество проверяющих и др. Отдельной задачей является указание критериев оценивания, их весов и баллов для оценивания.

2. Фаза представления работ. Студенты должны предоставить выполненные работы, загрузив их в систему. После этого происходит распределение работ для проверки между студентами. В зависимости от настроек Семинара распределение может происходить как случайным образом, так и осуществляться вручную преподавателем. Количество работ для взаимопроверки также должно быть обозначено заранее при создании рассматриваемого элемента курса.

3. Фаза оценивания. В режиме экспертной оценки обучающиеся осуществляют рецензирование присланных работ. В этой фазе предполагается формирование двух оценок обучающегося, первая за выполненную работу, которую он загрузил в систему, и вторая за оценивание в качестве рецензента.

Стоит отметить, что преподаватель может вручную переоценить работу студента или поучаствовать в качестве дополнительного рецензента.

4. Фаза оценивания оценок. Устанавливаются параметры для вычисления результатов за оценивание полученных работ. Основная задача на этом этапе - рассчитать итоговые оценки для представленных материалов и оценок, а также обеспечить обратную связь для авторов и рецензентов. Также устанавливаются параметры отображения материалов в следующей фазе.

5. Фаза закрытия семинара. Эта фаза завершает выполнение взаимопроверки работ. Отображаются результаты взаимопроверки, а также оценка за оценивание. Студенты могут просматривать как присланные материалы, так и результаты оценивания другими студентами.

Очевидно, что при всех положительных возможностях модуля Семинар действия преподавателя для организации взаимопроверки по курсу занимают достаточно много времени, что часто останавливает преподавателей от использования такой технологии, как взаимопроверка работ студентов в своей преподавательской деятельности.

Это приводит к ситуации, когда в условиях необходимости внедрения новых деятельных, развивающих форм, средств и методов обучения и контроля результатов обучения, преподаватели отказываются от использования такого средства автоматизации взаимопроверки, как Семинар в LMS Moodle в силу существенных временных и трудозатрат. Следовательно, становится актуальным вопрос оптимизации процесса создания модуля Семинар.

\section{П^агин автоматизированной генерации заланий Семинаров в LMS Moodle}

Одним из решений автоматизации организации взаимопроверки является инструмент резервного копирования в LMS Moodle путем экспорта и последующего импорта данных в файлах.mbz. При этом следует отметить следующие особенности:

- необходимо после импорта экземпляра экспортированного ранее Семинара вносить изменения, в частности, исходного задания, инструкций по выполнению заданий и критериев оценивания, а также соответствующих файлов медиаданных (например, образцы корректного выполнения работы, инструкции по проверке заданий при взаимопроверке, трактовку критериев и т.д.);

- преподавателю необходимо оценивать степень схожести созданных Семинаров по соответствующей тематике, количество критериев оценивания и т.д.

Таким образом, несмотря на то, что указанный подход способен оптимизировать процесс создания заданий для взаимопроверки. Он также не снимает в полной мере актуальность вопроса оптимизации временных затрат на создание заданий для взаимопроверки. 


\begin{tabular}{|c|c|c|c|}
\hline Название поля & Тип поля & Обязательно & Описание поля \\
\hline Название & Є Текстовое поле & Да & Название задания \\
\hline Описание & А Текстовая область & Да & Описание семинара \\
\hline Исходные материалы & Поле «Файл» & Да & Файл, который необходимо ... \\
\hline Инструкция & A Текстовая область & Да & Общие указания по оценке ... \\
\hline Образец & Поле «Файл» & Да & Привер выполнения работы С ... \\
\hline
\end{tabular}

Рис. 2. Поля базы данных «Задания»

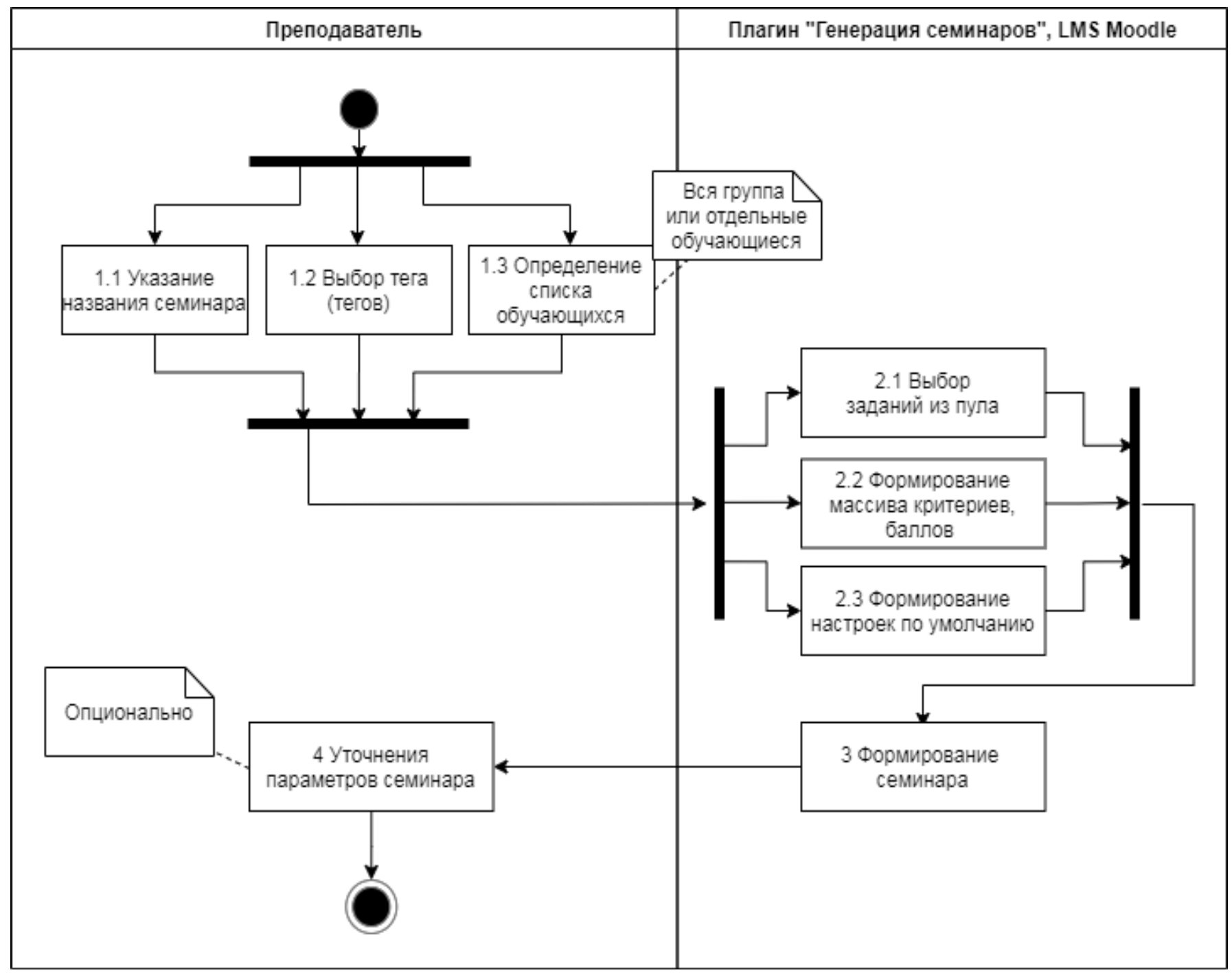

Рис. 3. Диаграмма активности плагина автоматизированной генерации задания Семинара 


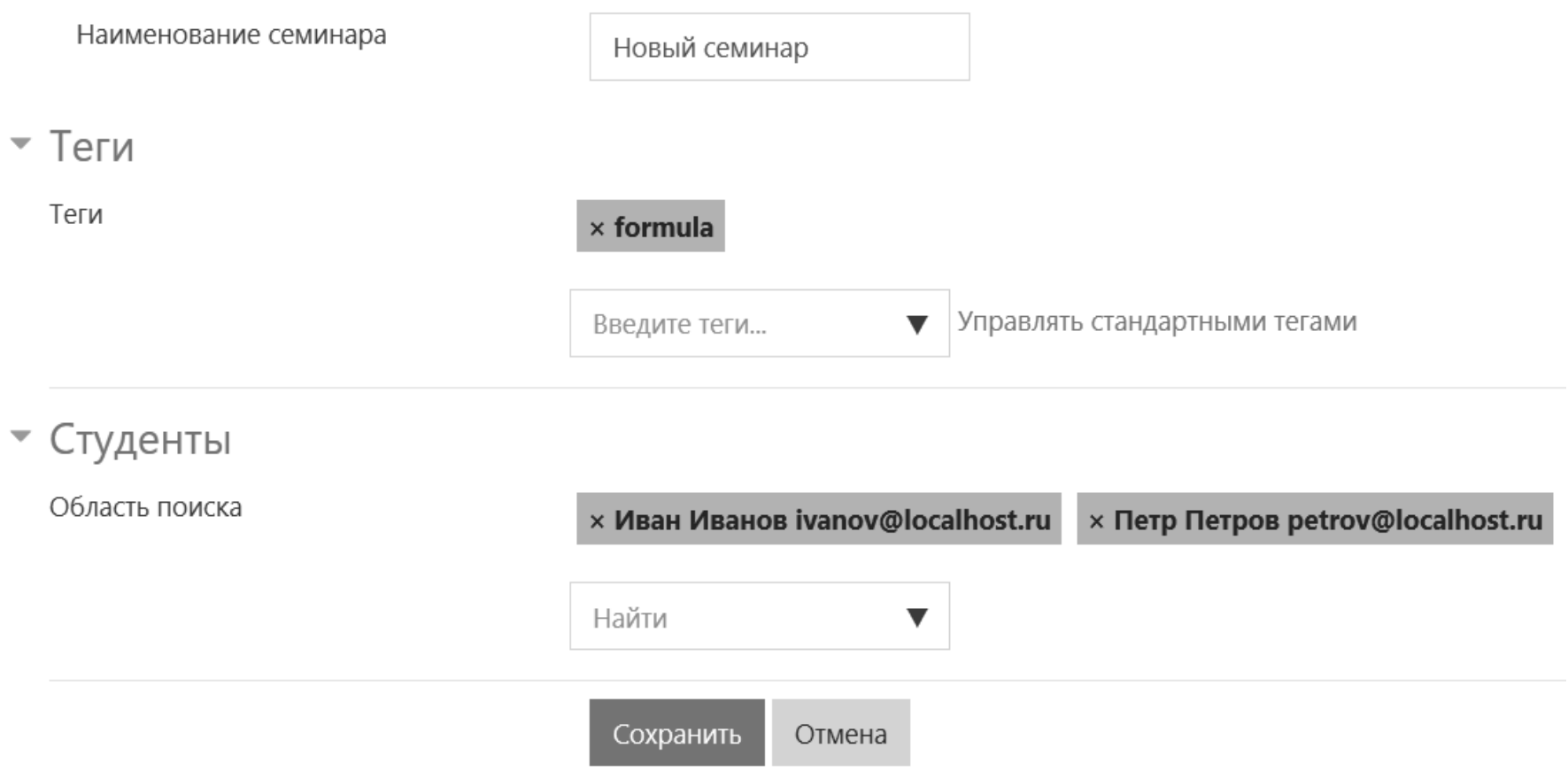

Рис. 4. Интерфейс плагина «Генерация семинаров»

Для решения проблемы оптимизации процесса создания модулей Семинар предлагается использовать альтернативный подход - разработка плагина автоматизированной генерации Семинаров в LMS Moodle.

В ходе исследования был спроектирован и реализован плагин для LMS Moodle, позволяющий автоматизировать процесс создания Семинаров на основе загруженных ранее данных.

В основе работы плагина, автоматизирующего создание модулей Семинар и распределение заданий для взаимопроверки, лежит понятие «тег». Под тегом рассматривается условное обозначение типичных задач в рамках выполнения задания, направленных на приобретение возможности осуществлять необходимое действие, не делая ее выполнение своей сознательной целью [10].

В частности, в статье [10] указано, что использование тегов в заданиях и описаниях подзадач позволяет:

- выявлять пробелы в знаниях и умениях студента,

- исключать повторения действий, которые освоены и не вызывают сложности в их выполнении обучающимися,

- определять сочетания типичных задач, которые вызывают затруднения в освоении,

- в несколько раз снизить затрачиваемое преподавателем время на разработку задания и критериев оценивания и др.
Для работы плагина были созданы две базы данных (с использованием соответствующего стандартного элемента LMS Moodle) «Критерии» и «Задания». База данных «Критерии» содержит такие поля, как тег, описание критерия, инструкция для выполнения соответствующих заданий модуля Семинар, инструкции для оценивающих, максимальная оценка, вес и файл с дополнительной информацией. Структура базы данных «Задания» и ее отображение в LMS Moodle представлена на рисунке 2.

На рисунке 3 представлена диаграмма активности автоматизированного создания заданий плагином «Генерация семинаров» LMS Moodle.

Как представлено на рисунке 3, на этапах 1.1-1.3 необходимо указать название Семинара, теги, по которым будет проводиться поиск в базах данных «Задания» и «Критерии», а также выбрать из числа обучающихся, записанных на курс, тех, кому будет доступен этот $\mathrm{Ce}$ минар. В случае, если не выбран ни один из студентов, созданный Семинар будет доступен всем обучающимся курса.

Пример заполненной формы представлен на рисунке 4.

С целью индивидуализации образовательного процесса создание заданий проводится на основе результатов, продемонстрированных обучающимся ранее. Так 
В плагине «Генерация семинаров» реализована функциональная возможность отображения статистических данных по выполнению заданий Семинаров по соответствующим тегам. В частности, по каждому обучающемуся на курсе доступны следующие данные по каждому тегу:

- количество работ, выполненных обучающимся,

- максимальное количество баллов, которое можно было набрать в выполненных работах в результате взаимопроверки,

- количество набранных баллов в ходе взаимопроверки,

- процентное отношение набранных баллов к теоретически возможному значению суммы баллов,

- вес тега.

На этапах 2.1-2.2, указанных на рисунке 3, происходит выбор исходных данных для создаваемого модуля Семинар в соответствии с указанными тегами. Поиск осуществляется по тегам LMS Moodle, которые отмечены как стандартные. Выбор студентов, для которых происходит подбор заданий, происходит с помощью соответствующего механизма LMS Moodle, а ограничение доступа осуществляется по адресу электронной почты обучающегося.

Например, исходные данные для обучения оформлению текстовой учебной документации (например, отчетов по НИР) могут быть представлены в виде файлов docх, в которых может требоваться восстановить и/или исправить форматирование элементов. В этих текстовых документах могут содержаться задания, соответствующие различным тегам. В этом случае обучающемуся предоставляется один из файлов с исходным заданием по указанным тегам, а в инструкции к выполнению автоматически указывается, какие действия необходимо выполнить.

На этапе 2.3 происходит определение значений $\mathrm{Ce}$ минара по умолчанию.

После этого происходит генерация элемента модуля Семинар с учетом указанных ранее параметров (этап 3 на рисунке 3). На завершающем этапе преподаватель имеет возможность при необходимости внести коррективы в параметры по умолчанию, например, временно скрыть элемент от обучающихся, указать даты основных фаз, задать параметры группового режима, предоставить дополнительную информацию по выполнению и оцениванию работ и т.д.

Стоит отметить, что предлагаемый плагин целесообразно применять в случае, когда заранее определены типовые параметры Семинара, а также заранее сформирован пул заданий с указанием соответствующих тегов и критериев оценивания с указанием тега, веса, баллов при оценивании.
Таким образом, разработанный плагин позволяет сократить время преподавателя, затрачиваемое на рутинную работу за счет заранее предустановленных параметров и определенных тегов. При этом преподаватель работает со стандартными средствами LMS Moodle и в привычном интерфейсе.

\section{Зак^ючение}

Показано, что взаимопроверка студенческих работ является перспективной и актуальной технологией организации процесса обучения в условиях распространения и повышения значимости дистанционного и смешанного обучения. Взаимопроверка является предпочтительным способом проверки работ студентов, когда работ очень много, и когда возможна автоматизация этого процесса.

Раскрыта необходимость автоматизации рутинных и требующих существенных временных затрат действий по организации взаимопроверки работ обучающихся.

В качестве средства, позволяющего снизить временные затраты преподавателя и упростить его работу по организации взаимопроверки в модуле Семинар LMS Moodle, предложен плагин. Использование разработанного плагина позволяет:

1. создавать Семинары с заранее предустановленными параметрами, указав только теги, по которым происходит подбор задания;

2. создавать Семинары с возможностью устанавливать обучающихся или группу обучающихся, для которых подобранное задание будет актуальным;

3. изменять стандартными средствами LMS Moodle параметры созданных Семинаров.

При этом работа по настройке параметров, добавлению данных ведется с использованием стандартных элементов курса База данных и Семинар, а интерфейс разработанного плагина соответствует интерфейсу LMS Moodle.

Разработанный плагин готов к внедрению, с его помощью можно:

- оптимизировать деятельность преподавателя за счет сокращения количества рутинных действий,

- экономить ресурсы на создание подзадач и критериев оценки,

- определять более точно выбор соответствующих актуальных заданий,

- минимизировать ошибки в процессе подготовки заданий для обучающихся. 


\section{ЛИТЕРАТУРА}

1. Trautmann, N. M. Interactive learning through web-mediated peer review of student science reports. Educational Technology Research and Development, 2007. 57(5), 685-704.

2. Гоголь А.А., Томашевич С. В., Красов А. В., Сетевой метод взаимопроверки знаний студентов // Материалы Х всероссийской (с международным участием) научно-практической конференции. - 2008. - С. 222-226.

3. Смирнова 0.Б., Приходько М. А., Долгова Л. В. Об организации взаимопроверки в условиях промежуточного контроля по математике // Актуальные вопросы образования и науки. - 2019.— № 2. - C. 55-58.

4. L. de Alfaro and M. Shavlovsky. CrowdGrader: A tool for crowdsourcing the evaluation of homework assignments. In Proceedings of the 45th ACM technical symposium on Computer science education, pages 415-420. ACM, 2014.

5. Информационные и коммуникационные технологии в образовании: монография / Под редакцией Бадарча Дендева. М.: ИИТО ЮНЕСКО, 2013.

6. Лесин С.М., Махотин Д. А. МООС как современная технология организации смешанного обучения // Интерактивное образование. 2018, № 1-2, С. 25-34.

7. Дронова Е. Н. Технологии дистанционного обучения в высшей школе: опыт и трудности использования // Преподаватель XXI Век — 2018. - № 3-1. C. 23-34

8. Анисимов А. М. Работа в системе дистанционного обучения Moodle. Учебное пособие. — Харьков, ХНАГХ, 2008. — 275 стр. С. $117-127$.

9. Using Workshop — Moodledocs: [caйт]. URL: https://docs.moodle.org/38/en/Using_Workshop (дата обращения 12.06.20).

10. Насыров Н.Ф., Кобец Е. А., Горлушкина Н. Н. Автоматизированная генерация учебных подзадач на основе методики тегов и критериев // Современная наука: актуальные проблемы теории и практики. Серия «Естественные и технические науки» — 2020.— № 3.— С. 102-107

( Насыров Наиль Фаизович ( pasdel@mail.ru ), Горлушкина Наталия Николаевна ( nagor.spb@mail.ru ),

Валитова Юлия Олеговна ( julijawal@gmail.com ), Тартынских Петр Сергеевич ( tartynskikh.ps@yandex.ru ).

Журнал «Современная наука: актуальные проблемы теории и практики»

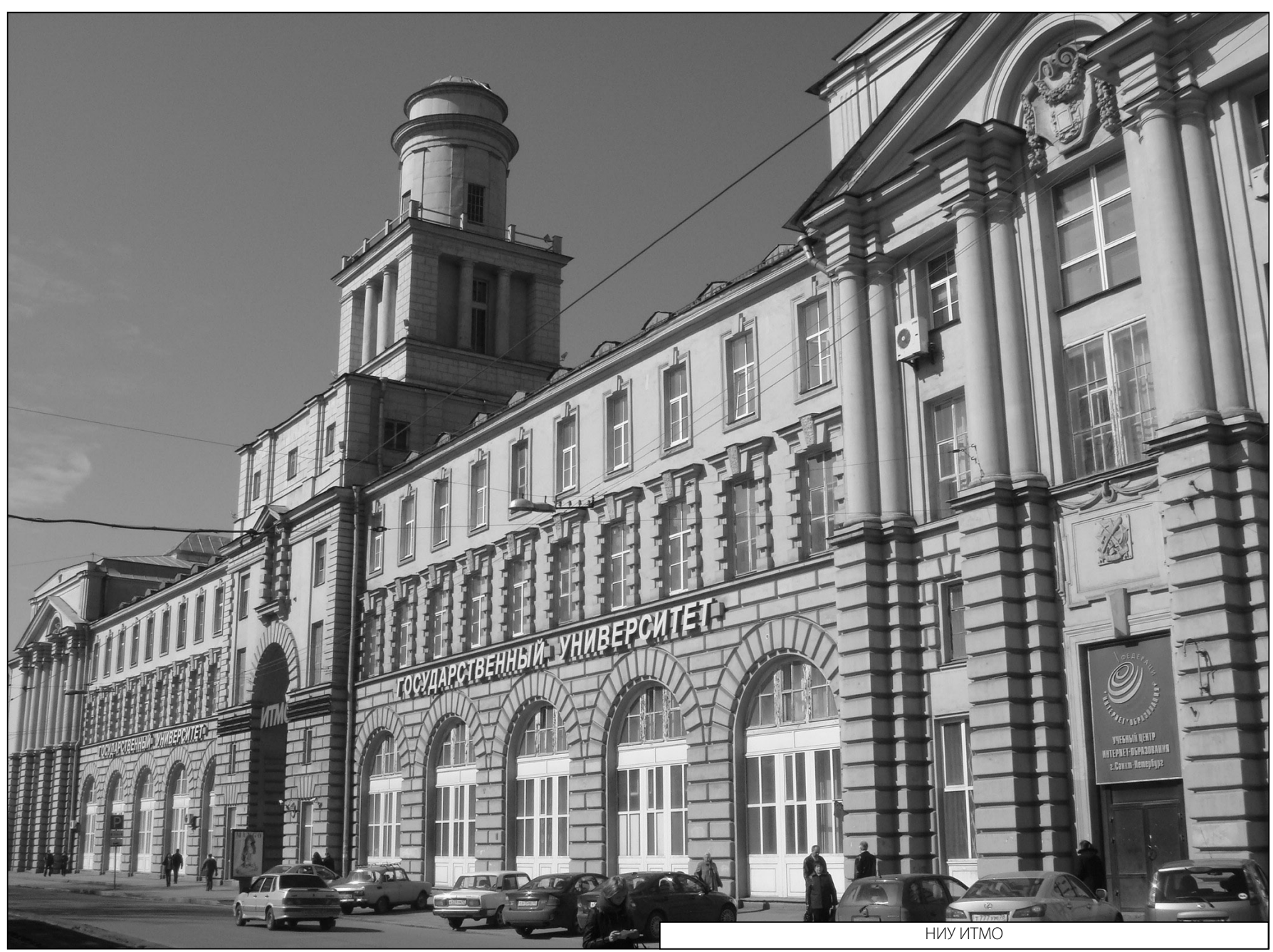

